\title{
THE SYMMETRY OF THE WEIERSTRASS GENERALIZED SEMIGROUPS AND AFFINE EMBEDDINGS
}

\author{
FELIX DELGADO \\ (Communicated by Louis J. Ratliff, Jr.)
}

\begin{abstract}
The characterization of the property of complete intersection for affine curves with one place at infinity in terms of the Weierstrass semigroup can be generalized to the case of several places at infinity.
\end{abstract}

\section{INTRODUCTION}

Let $X$ be a locally complete intersection projective curve of arithmetical genus $g$ over an algebraically closed field $k$. For each regular point $P \in X(P \in$ $X_{\text {reg }}$ for short ) denote by $v_{p}$ the discrete valuation of $K(X)$ associated to $P$. Let $P_{1}, \ldots, P_{d} \in X_{\text {reg }}$, and set $v_{i}=v_{p_{i}}$. One can consider the affine coordinate ring of $X^{\prime}=X-\left\{P_{1}, \ldots, P_{d}\right\}, A=\Gamma\left(X^{\prime}, \mathscr{O}_{X}\right)$ and, on the other hand, the generalized Weierstrass semigroup for the set of points $\left\{P_{1}, \ldots, P_{d}\right\}$ given by

$$
\Gamma=\left\{-\left(v_{1}(f), \ldots, v_{d}(f)\right) \mid f \in A\right\} .
$$

If $d=1, \Gamma$ is the usual Weierstrass semigroup for $P_{1}$. In [7] Sathaye proves that $X-\left\{P_{1}\right\}$ is a complete intersection affine curve if and only if $\Gamma$ is a symmetrical semigroup with respect to the element $2 g-1$. This fact gives an analogy with the symmetry property proved by Kunz in [5] for the characterization of the Gorenstein curve singularities in terms of its semigroup of values. In fact $c=2 g$ becomes the conductor for such a Weierstrass semigroup and $c-1=2 g-1$.

In this paper we will prove that the result by Sathaye is also true for $d>1$ if one considers the concept of symmetry analogous to that given in our previous paper [3] for subsemigroups of $\mathbf{N}^{d}$ associated with reduced curve singularities. In [3] it is shown how the symmetry of the semigroup is equivalent to the singularity being Gorenstein.

Received by the editors September 16, 1988 and, in revised form, April 7, 1989.

1980 Mathematics Subject Classification (1985 Revision). Primary 14H05; Secondary 14E25.

Key words and phrases. Weierstrass semigroups, affine complete intersections.

Partially supported by the CICYT no. 336/87. 
Note that in [1, VIII], the Weierstrass semigroup of $P_{1}, \ldots, P_{d}$ is defined in a different fashion, as follows:

$$
\widetilde{\Gamma}=\left\{\left(n_{1}, \ldots, n_{d}\right) \in \mathbf{Z}^{d} \mid \exists f \in K(X),(f)_{\infty}=\sum n_{i} P_{i}\right\},
$$

so one has $\tilde{\Gamma}=\Gamma \cap \mathbf{N}^{d}$.

Notations. Let $n=\left(n_{1}, \ldots, n_{d}\right) \in \mathbf{Z}^{d}$ and $J=\left\{i_{1}, \ldots, i_{r}\right\} \subset I=\{1, \ldots, d\}$. We will use the following notations:

$$
\begin{aligned}
\bar{\nabla}_{J}(n) & =\bar{\nabla}_{i_{1} \ldots i_{r}},(n)=\left\{m \in \mathbf{Z}^{d} \mid m_{i}=n_{i} \forall i \in J, m_{j}<n_{j} \forall j \notin J\right\}, \\
\bar{\nabla}(n) & =\bigcup_{i=1}^{d} \bar{\nabla}_{i}(n) ; \quad \nabla_{J}(n)=\bar{\nabla}_{J}(n) \cap \Gamma ;
\end{aligned}
$$

and

$$
\begin{gathered}
\nabla(n)=\bar{\nabla}(n) \cap \Gamma=\left\{m \in \Gamma \mid m_{j}<n_{j} \forall j, \text { and } m_{i}=n_{i} \text { for exactly one } i\right\}, \\
\nabla_{i}^{d}(n)=\bigcup_{i \in J} \nabla_{J}(n)=\left\{m \in \Gamma \mid m_{i}=n_{i} ; m_{j} \leq n_{j} \forall j \neq i\right\} .
\end{gathered}
$$

Remark. Assume that $X$ is a projective curve of arithmetical genus $g$ and denote by $X_{\text {reg }}$ the set of regular points of $X$. For each invertible sheaf $\mathscr{L}$ on $X$ there exists a divisor $D=\sum_{1}^{t} n_{i} Q_{i}$ with support in $X_{\text {reg }}$, i.e., $Q_{i} \in X_{\text {reg }}$ for all $i$, in such a way that $\mathscr{L}=\mathscr{L}(D)$. In particular, if we assume that $X$ is a locally complete intersection the dualizing sheaf $\omega_{X}$ of $X$ is an invertible sheaf on $X$ and we can take the canonical divisor $K$ to be a divisor with support in $X_{\text {reg }}$ corresponding to $\omega_{X}$. In this case one also has the corresponding Riemann-Roch formula:

$$
\ell(D)-\ell(K-D)=\operatorname{deg}(D)+1-g .
$$

If $D=\left\{\left(U_{i}, f_{i}\right)\right\}_{i \in I}$ is a Cartier divisor in $X$ and $P \in X_{\text {reg }}$, we will denote $v_{p}(D)$ the value $v_{p}\left(f_{j}\right)$ if $P \in U_{j}$. We will also use the usual notations to denote the effectivity of $D(D>0)$, and the linear equivalency between two divisors $\left(D \sim D^{\prime}\right)$.

As in the introduction, let $P_{1}, \ldots, P_{d} \in X_{\text {reg }}$; if $n=\left(n_{1}, \ldots, n_{d}\right) \in \mathbf{Z}^{d}$ consider the divisor $D(n)=\sum_{1}^{d} n_{i} P_{i}$, the invertible sheaf $\mathscr{L}(D(n))$ and the vector space of global sections of $\mathscr{L}(D(n))$ denoted here by $\Gamma(X, n)$. Also set $\ell(n)=\operatorname{dim}_{k} \Gamma(X, n)$ and $\ell(K-n)=\operatorname{dim}_{k} \Gamma(X, \mathscr{L}(K-D(n)))$. The vector space $\Gamma(X, n)$ can be seen as the set $\Gamma(X, n)=\{f \in K(X) \mid(f)+D>0\}$ $=\left\{f \in A \mid v_{i}(f) \geq-n_{i}, i=1, \ldots, d\right\}$. Finally, we will denote by $\mathbf{e}_{1}, \ldots, \mathbf{e}_{d}$ the standard basis of $\mathbf{Z}^{d}$, i.e., $\mathbf{e}_{i}=(0, \ldots, 0,1,0, \ldots, 0) \in \mathbf{Z}^{d}$ ( 1 in the $i$ th place).

Definition. Let $n \in \mathbf{Z}^{d}$, then $n$ will be called a relative maximal for $\Gamma$ if $\nabla(n)=\varnothing$ and if for any $J \subset I$ with $\# J \geq 2$ one has $\nabla_{J}(n) \neq \varnothing$. 
Definition. The semigroup $\Gamma$ will be said to be symmetric with respect to $\tau$ if it satisfies the following property:

$$
\text { "If } n \in \mathbf{Z}^{d} \text { then } n \in \Gamma \text { if and only if } \nabla(\tau-n)=\varnothing \text { ". }
$$

Remark. The nature of the Weierstrass semigroups is different than that of the semigroups associated with curve singularities studied in [2] and [3]. However if one considers $-\Gamma=\left\{\left(v_{1}(f), \ldots, v_{d}(f)\right) \mid f \in A\right\}$ (in some aspects more natural than $\Gamma$ ) then the sets $\bar{\Delta}_{J}, \Delta_{J}, \Delta, \ldots$ considered in $[2,3]$ are related to $\bar{\nabla}_{J}, \nabla_{J}, \nabla$ for the semigroup $\Gamma$, as follows:

$$
\triangle_{J}(-n)=-\bar{\nabla}_{J}(n), \quad \Delta_{J}(-n)=\nabla_{J}(n), \ldots \quad \forall n \in \mathbf{Z}^{d} .
$$

This fact motivates the above definitions. Thus these concepts of symmetry and maximals are the same as in [3].

Remark. The Weierstrass semigroup $\Gamma$ satisfies analogous properties to those given in $[2,(1.2)]$ or $[3,(1.1)]$ for the semigroups of values of curve singularities. Namely one has:

-If $n, m \in \Gamma$, then $\operatorname{Sup}(n, m)=\left(\max \left\{n_{1}, m_{1}\right\}, \ldots, \max \left\{n_{d}, m_{d}\right\}\right) \in \Gamma$.

-If $n, m \in \Gamma$ with $n_{i}=m_{i}$ for some $i \in I$, then there exists $c \in \Gamma$ such that $c_{r} \leq \max \left\{n_{r}, m_{r}\right\} \forall r \in I$ (and the equality holds if $n_{r} \neq m_{r}$ ) and $c_{i}<n_{i}=m_{i}$.

On the other hand, using the Riemann-Roch Theorem one finds other properties rather different than those of the semigroups of values, as for instance the following one:

-If $n \in \mathbf{Z}^{d}$ is such that $\sum n_{i} \geq 2 g$ then $n \in \Gamma$ and if $\sum n_{i}<0$ then $n \notin \Gamma$.

In the same way as in [2] or [3] one can prove also the corresponding generation theorem:

Theorem. Let $m \in \mathbf{Z}^{d}$; then $m \notin \Gamma$ if and only if there exists a relative maximal $n$ of $\Gamma$ such that $m \in \bar{\nabla}(n)$.

However, note that in this case the set of relative maximals is always infinite.

Proposition. With notations as above, if $n=\left(n_{1}, \ldots, n_{d}\right) \in \mathbf{Z}^{d}$ then one has:

(i) $n \in \Gamma$ if and only if $\ell(n)=\ell\left(n-\mathbf{e}_{i}\right)+1 \forall i=1, \ldots, d$.

(ii) $\nabla_{i}^{d}(n)=\varnothing$ if and only if $\ell(n)=\ell\left(n-\mathbf{e}_{i}\right)$.

(iii) $\nabla(n)=\varnothing$ if and only if there exists a divisor $K^{\prime} \sim K$ on $X$ such that $v_{i}\left(K^{\prime}\right)=n_{i}-1, \forall i=1, \ldots, d$ and $K^{\prime}-\sum\left(n_{i}-1\right) P_{i}>0$.

Proof. The first statement is clear, because $n \in \Gamma$ if and only if there exists $f \in A$ such that $-v_{i}(f)=n_{i}$ for every $i \in I$, so $f \in \Gamma(X, n), f \notin$ $\Gamma\left(X, n-\mathbf{e}_{i}\right)$ and hence $\ell\left(n-\mathbf{e}_{i}\right)=\ell(n)-1$ for every $i \in I$. Conversely, if there exists $f_{i} \in \Gamma(X, n)-\Gamma\left(X, n-\mathbf{e}_{i}\right) \forall i=1, \ldots, d$, then taking general elements $\lambda_{1}, \ldots, \lambda_{d} \in k$ one finds $f=\sum \lambda_{i} f_{i} \in A$ such that $f \in \Gamma(X, n)-\Gamma\left(X, n-\mathbf{e}_{i}\right)$ for every $i$, so $v_{i}(f)=-n_{i}$ and $n \in \Gamma$. 
For the second statement, note that $\ell(n)=\ell\left(n-\mathbf{e}_{i}\right)+1$ if and only if there exists $f \in \Gamma(X, n)-\Gamma\left(X, n-\mathbf{e}_{i}\right)$ and this is equivalent to $-\left(v_{1}(f), \ldots\right.$, $\left.v_{d}(f)\right) \in \nabla_{i}^{d}(n)$.

For the last statement one has the following equivalences:

$$
\begin{aligned}
\nabla(n)=\varnothing & \Longleftrightarrow \nabla_{i}^{d}\left(n-\sum_{j \neq i} \mathbf{e}_{j}\right)=\varnothing, \forall i \in I \\
& \Longleftrightarrow \ell\left(n-\sum_{j \neq i} \mathbf{e}_{j}\right)=\ell\left(n-\sum_{j=1}^{d} \mathbf{e}_{j}\right) \forall i \in I \\
& \Longleftrightarrow \ell\left(K-n+\sum_{j \neq i} \mathbf{e}_{j}\right)+1=\ell\left(K-n+\sum \mathbf{e}_{j}\right), \forall i \in I \\
& \Longleftrightarrow \exists f \in \Gamma\left(X, K-n+\sum \mathbf{e}_{j}\right)-\Gamma\left(X, K-n+\sum_{j \neq i} \mathbf{e}_{j}\right), \forall i .
\end{aligned}
$$

This statement is equivalent to the existence of a divisor $K^{\prime} \sim K$, namely $K^{\prime}=(f)+K$, in such a way that $v_{i}\left(K^{\prime}\right)=n_{i}-1$ and $K^{\prime}-D(n-1)>0$.

Theorem. Let $X$ be a locally complete intersection projective curve of arithmetical genus $g$ and $X^{\prime}=X-\left\{P_{1}, \ldots, P_{d}\right\}$, where $P_{i} \in X_{\text {reg }}, \forall i=1, \ldots, d$. Then the following conditions are equivalent:

(1) $X^{\prime}$ is an affine complete intersection.

(2) There exists a canonical divisor $K$ such that $\operatorname{supp}(K) \subset\left\{P_{1}, \ldots, P_{d}\right\}$.

(3) There exists a relative maximal $\tau=\left(\tau_{1}, \ldots, \tau_{d}\right)$ in $\Gamma$ such that $\sum \tau_{i}=$ $2 g-2+d$.

(4) There exists $\sigma=\left(\sigma_{1}, \ldots, \sigma_{d}\right) \in \Gamma$ such that $\sum \sigma_{i}=2 g-2+d$ and $\Gamma$ is symmetrical with respect to $\sigma$.

Proof. The equivalence between (1) and (2) is a straightforward generalization of the results in [6] (see also [7]) taking into account the corresponding ones by Serre in [8]. On the other hand, the equivalence between (2) and (3) results from part (iii) in the above Proposition.

Assume that there exists a relative maximal $\tau$ in $\Gamma$ such that $\sum \tau_{i}=$ $2 g-2+d$. We will prove that $\Gamma$ is symmetrical with respect to $\tau$. If $n \in \Gamma$ then $\nabla(\tau-n)=\varnothing$ since otherwise, if one takes $m \in \nabla(\tau-n)$ then one would have $m+n \in \nabla(\tau)$, which is a contradiction. Conversely, assume $\nabla(\tau-n)=$ $\varnothing$, and take a canonical divisor $K$ such that $\operatorname{supp}(K) \subset\left\{P_{1}, \ldots, P_{d}\right\}$ and $v_{i}(K)=\tau_{i}-1$ for any $i \in I$. One can also take (by (iii) of the above Proposition) $K^{\prime} \sim K$ such that $v_{i}\left(K^{\prime}\right)=\tau_{i}-n_{i}-1 \forall i$ and $K^{\prime}-D(\tau-n-1)>0$. Thus $K^{\prime}=(f)+K$ with $f \in K(X)$. Now, the divisor $K$ is given in the open $X^{\prime}=X-\left\{P_{1}, \ldots, P_{d}\right\}$ by $1 \in \Gamma\left(X^{\prime}, \mathscr{O}_{X}\right)$ so $K^{\prime}$ is given in the same open by $\left(X^{\prime}, f\right)$, but $K^{\prime}-D(\tau-n-1)>0$ implies in this case $f \in \Gamma\left(X^{\prime}, \mathscr{O}_{X}\right)=A$. Since for any $i$ one has $-v_{i}(f)=v_{i}(K)-v_{i}\left(K^{\prime}\right)=\left(\tau_{i}-1\right)-\left(\tau_{i}-n_{i}-1\right)=n_{i}$, one gets $n \in \Gamma$. This proves $(3) \Rightarrow(4)$. 
Now, assume that there exists $\sigma=\left(\sigma_{1}, \ldots, \sigma_{d}\right) \in \Gamma$ such that $\Gamma$ is symmetrical with respect to $\sigma$ and $\sum \sigma_{i}=2 g-2+d$. One must only prove that $\nabla(\sigma)=\varnothing$, because the condition $\nabla_{J}(\sigma) \neq \varnothing$ for any $J \subset I$ with $\# J \geq 2$ is trivially satisfied. For this, assume that there exists $n \in \nabla(\sigma)$; then $\nabla(\sigma-n)=\varnothing$ which is contradictory to the fact $0 \in \nabla(\sigma-n)$. This completes the proof of the theorem.

Remark. Note that statement (3) in the above theorem does not provide a finite procedure to test if the affine curve $X^{\prime}=X-\left\{P_{1}, \ldots, P_{d}\right\}$ is a complete intersection because the number of elements $\tau$ in $\Gamma$ such that $\sum \tau_{i}=2 g-2+d$ is not finite. In this sense it would be interesting to give an answer to the following question: Does there exist a natural number $T$ (depending on the genus and the number $d$ ) such that $X^{\prime}$ is an affine complete intersection if and only if there exists a relative maximal $\tau$ in $\Gamma$ such that $\sum \tau_{i}=2 g-2+d$ and $\sum\left|\tau_{i}\right| \leq T$ ? Note that the above question can be stated in terms of a canonical divisor or, for smooth curves in terms of a differential form on the curve.

\section{REFERENCES}

1. E. Arbarello, M. Cornalba, P. A. Griffiths and J. Harris, Geometry of algebraic curves, vol. I Grundlehren der Math. Wiss. 267, Springer-Verlag, New York, 1985.

2. F. Delgado, The semigroup of values of a curve singularity with several branches, Manuscripta Math. 59 (1987), 347-374.

3. 285-296.

4. R. Harsthorne, Algebraic geometry, GTM no. 52, Springer-Verlag, Berlin, Heidelberg and New York, 1977.

5. E. Kunz, The value-semigroup of a one-dimensional Gorenstein ring, Proc. of the Amer. Math. Soc. 25 (1970) 748-751.

6. M. P. Murthy and J. Towber, Algebraic vector bundles over $\mathbf{A}^{3}$ are trivial, Invent. Math. 24 (1974) 173-189.

7. A. Sathaye, On planar curves, Am. J. Math. 99 (1974), 1105-1135.

8. J. P. Serre, Sur les modules projectifs, Seminaire Dubreil-Pisot 1960/61, No. 1.

Departmento de Algebra, Facultad de Matemáticas, Universidad Complutense, 28040-MADRID, SPAIN 